\title{
Emergence as a Subject of Research, Research Methods, and Engineering Knowledge and Practice
}

\author{
Timothy L.J. Ferris \\ Centre for Systems Engineering \\ Cranfield University, Defence Academy of the United Kingdom \\ Shrivenham, SN6 8LA, United Kingdom
}

\begin{abstract}
The paper discusses several views on the definition of the term "emergence" in relation to systems. The paper then discusses several approaches to research which have different purposes in order to identify the relationship of the research approach and the issue of emergence. Through this discussion it is possible to identify the relationship between research methodologies, engineering development and the issue of emergence. In particular, it is shown that the problem of emergence contains the core of a pathway to the engineering of systems.
\end{abstract}

Keywords-emergence; engineering; research; research methods

\section{INTRODUCTION}

The question we address in this paper is the interaction of the concept of emergence and research in the context of engineering. The concerns of system of systems engineering, SoSE, and systems engineering, SE, bring the challenges of research in engineering to the fore. In SoSE and SE there is explicit acknowledgement of the matter of emergence, in which the system resulting from the engineering work evidences characteristics which are not present in the elements of which the system or system of system is comprised.

Emergence is important in all engineering because the purpose of engineering work is to make useful products or systems which enable action that is not possible without he designed entity, and the designed entity enables the performance of the desired functions which were not achievable through other means.

The classical epistemological account of knowledge is expressed in the trifold conditional statement, or a variation: " $S$ knows that $p$ if and only if: $1 . p$ is true; $2 . S$ believes that $p$; and 3. $S$ is is justified in believing that $p$ " [1]. Gettier's paper is one of the most controversial papers in epistemology because he challenged this account of the requisites of knowledge and several variants but, in turn, many epistemologists have objected to his view. The next discussion of knowledge in engineering is predicated on the standard formulation of knowledge and the discussion of research approaches that follows, Section III, is built on constructs that the tradition of science has made upon this foundation.

Knowledge in engineering, from the perspective of a practicing engineer, concerns the behaviours of things that may be applied in technical solutions to issues, combined with knowledge of the contexts into which engineered things will be deployed in order to make useful and desirable contributions in the field of application. The engineer needs reliable knowledge of the factors, of all types, relevant to their project to enable an apposite vision of both what is to be achieved and the potential means for achieving that outcome. The methods of research are necessary to generate this knowledge and to provide assurance of its veracity.

The knowledge the engineer needs for practice differs from the knowledge traditionally developed in the many scientific disciplines, including the engineering science research which forms a large part of the university research in engineering departments. The latter kind of knowledge is focused on identifying and describing the relationships of phenomena, most usually abstracted from their contexts. Thus, each research project typically explores and reports the relationship of two manifestations abstracted from their context. In aggregate, a series of investigations may lead to a set of results that close a network of possible relationships and may enable the development of a theory that powerfully connects a large space, but still has the limitation of being a knowledge construct that abstracts that phenomenon from its broader context. For example, the history of the development of Maxwell's equations for electromagnetism is an example of such a development of theory, and it remains to explore the interaction of that theoretical construct with additional environmental conditions.

While, if one knew theoretical models to link all of the phenomena which may interact when designing a solution for a need, it may be possible to construct a model of the intended whole that accounts for the impact of all the possible effects, such a model is challenged in several ways, the difficulty of its construction, the potential absence of knowledge of some potential interactions, the computational difficulty of using the model to inform design, and the difficulty of obtaining data to describe its elements. That is, with full enough knowledge and resources to do the work many emergent effects may be discoverable but gaps in theoretical knowledge and other practical difficulties provide limits on attempting to predict emergent effects through analysis of standard scientific knowledge. In addition, there is the theoretical problem that one does not, and cannot, know whether one has knowledge of all relevant factors. 


\section{EMERGENCE}

The concept of emergence is somewhat problematic in systems engineering. A discussion of the matter may either produce recitation of simplistic statements, such as "the whole is greater than the sum of the parts", or an argument that emergence refers to the nasty surprises, referred to by some as "undocumented features", or, at least, that emergence refers to unexpected, even if desirable outcomes. None of these approaches is helpful.

To assert that "the whole is greater than the sum of the parts" does not explain anything that is helpful to a person seeking to design a system that will provide desired services, performance or some other characteristic, whilst simultaneously not providing undesired or undesirable outcomes. The simplistic statement does not explain how to achieve the desired in a situation.

To regard emergence as concerned with the unexpected outcomes results in a creeping boundary of what constitutes emergence. If a system is developed, assembling a number of components, and is found to have certain properties in addition to those that are expected then, on the first occasion, this definition would assert that emergence is present. If a second system is assembled and the same effect is observed, the situation is no longer emergence because the builder should reasonably expect the observed outcome. At least in some circumstances the legal this idea may be argued, particularly if there is a claim that the system developer either made or allowed an undesirable outcome that followed a previously observed pattern.

To define emergence as concerned with the appearance of undesirable effects which were not expected trivialises the problem of emergence in two ways. The first is as discussed above, of associating the idea of emergence with that which was not actually predicted, or predictable, depending on the analyst's view, or of linking the idea of emergence with the negative constructs of undesired and undesirable. Linking emergence with undesired outcomes is problematic because an outcome not desired by one party may be desired by another, yet the assembly of components into a system is the same.

These approaches to defining emergence are unsatisfactory because they yield a shifting answer to the question of whether observation of a particular outcome, in the case of a particular system, is emergent or not, and by associating negative connotations with emergence overlook the fact that the purpose of all engineering work is to achieve effects arising from the completed system that are not present in the components of the system taken separately.

To be useful a definition of anything needs to:

1. Provide a terminology that has constant breadth of inclusion of cases

2. Address a class of something that is independent of connotations of value concerning the goodness or otherwise of the subject matter.

In this paper we define emergence as: "characteristics of a system that arise from the assembling of elements of the system into a system, which are not observed in any of the elements of the system viewed alone."

This definition has the desirable characteristics of:

1. Not ascribing a value judgment, good or bad, on anything described as emergent.

2. Providing a constant boundary between emergence and non-emergence, that holds regardless of the current state of knowledge.

3. Accommodating the primary goal of engineering, to make systems that satisfy needs through assembling components to produce effects that are not achieved by the components taken alone.

A further philosophical issue arises as to whether, if one knows certain facts, one also knows the logical consequences of those facts [2]. This issue is important in relation to emergence because emergence is the consequence of assembling a number of elements which have their own properties and interactions which result in effects different than those observable in the elements taken alone. The issue, then, is whether in adducing certain facts about the elements of a system, which through the interaction of the manifestations which the facts represent, it can be said that one is aware of the consequences of the facts known and their interactions. If one were to be aware of the interactions one would not be taken by surprise by the interaction of known effects of known entities. However, the evidence of experience is that surprises do happen, so that one may be aware of entities and their characteristics but not have immediate awareness of the characteristics of a system which is a compound of the set of entities. In general design relies on the consistency of matter, that is, that things and matter behave in a manner consistent with their properties, such that if the properties of a number of elements, when previously assembled in a certain way have resulted in observation of a certain set of outputs, then in future cases one can project that the same outputs would be observed if the same set of elements were assembled [3].

Checkland and Holwell [4] provided a relatively simple description of an academic discipline as involving a set of: a framework of ideas, and area of concern and a methodology for applying ideas in the framework to the area of concern. This model was added to, with the addition of a second methodology, the methodology for improving or updating the framework of ideas, because it was recognised that the framework of ideas may be improved through a method that differs from the method used for applying the discipline [5].

More recently Rousseau et al [6] have presented the Disciplinary Maturity, D-MAT, model, Fig 1. The D-MAT organises the issue of a discipline through asking a number of questions. The first three, Q1-Q3 concern the boundary of the discipline, what is included and what excluded. Q4 concerns description of the entities which are the subject matter of the discipline. The next question concerns how the entities work, and so has the first stage of theoretical development in the understanding of the discipline. The final four questions are closely related in kind, concerning how and why the entities which are the subject matter of the discipline come about and behave. The focus of the D-MAT is on a discipline as a kind of 
science, concerned with providing explanation of how and why the world is as it is. This contrasts with the possibility of the discipline being a kind of technology, a means to do something. Where a discipline is a technology the purpose of the science aspect of the discipline is to produce the knowledge which describes the facts of the case or cases in a form which makes those facts useful for understanding and theorising about relationships within the scope of the discipline and for informing prediction of what would happen if particular actions are taken. This work is critical in any field in which the purpose is to enable intervention with the purpose of achieving a particular goal.

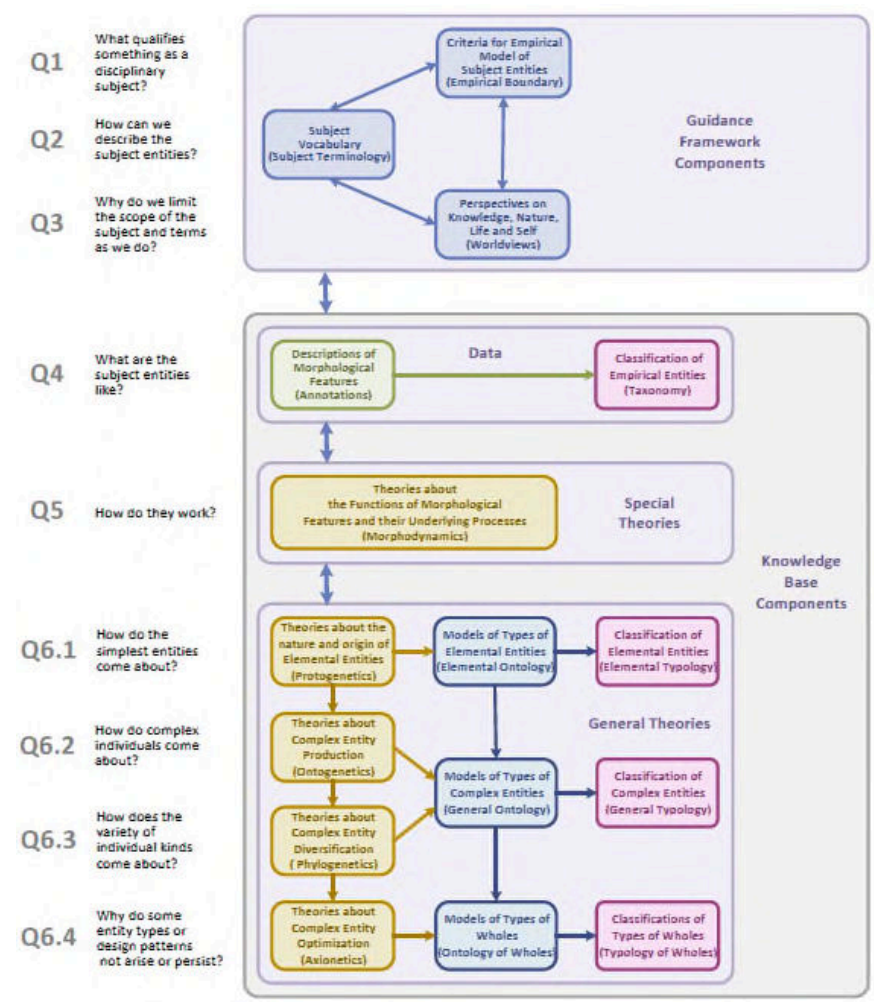

Fig. 1. The Discipline Maturity, D-MAT, model of Rousseau et al [6]

\section{TYPES OF RESEARCH USED TO INVESTIGATE EMERGENT SITUATIONS}

We discuss five classes of research activity which have relevance to emergence below.

\section{A. Case Studies}

Under the heading of case studies we refer to investigations of cases initiated by the researcher out of some kind of interest in discovering the facts and relationships in a case. As such a case study is conducted by a person who believes there is something interesting about a particular case and investigates by finding all the accessible materials, which could include previously published items, and primary sources and interviews with stakeholders and other similar things.
In relation to the question of emergence it is most likely that the case is known because something unusual happened and is attributed to "emergence" because the observed situation had not been expected.

A major limitation of a case study as an investigation method is that in an investigator interest driven situation the investigator can only obtain source materials that the participants with direct interaction with the system choose to make available. The impact of this selection of sources that the investigator is permitted or assisted to see is that the investigator may assume that there is no other view to be found in any materials not divulged. In the absence of any other information, the researcher may be left with questions concerning what other facts may be relevant to the situation, leading to limitations in the conclusions that can be drawn or the confidence in the veracity of those conclusions. A second major limitation is that the case study presents an account of a particular case which then raises the question of the extent to which the description of one particular case can and should be associated with a generalisation of that case to other cases. The problem is whether the case should be read as just a description of a past case or should be used as a recommendation for others in analogous situations to behave in a similar way.

Case studies are often performed in one of two scenarios. The first of these is the case study related to a disaster, usually on a notable scale that made the original story newsworthy. These scenarios are likely to identify emergence arising through the confluence of factors which had a dramatic impact. Often the lesson is drawn to deal with the specific kind of factor that the investigator identifies as a crucial contributor to the bad effect. The second common case study scenario is that which led to a good outcome, in which case the lesson, explicit or subliminal, is "go thou and do likewise".

In summary, case studies give post hoc accounts of cases selected by a researcher because the researcher believes the case may shed interesting light on a matter of interest to the researcher. The case study method looks at single cases from as many perspectives accessible to the researcher and so can provide some insight into emergence with caveats concerning the limitations of generalisability or other approaches to application of the learning.

\section{B. Forensic Investigations}

Forensic investigations are, like case studies, investigations of particular cases. However, they are conducted with a specific purpose of discovering the cause of some problem, either to attribute blame or to generate recommendations to avoid repetition. As such, forensic investigations are performed out of a need to fulfil an investigation of a matter apart from the investigator for a specific purpose, either legal or organisational. Since the forensic investigation is conducted to make discoveries about the cause of a problem or to make recommendations of methods to prevent future similar difficulties they are conducted with either legal or organisational persuasive powers to enforce cooperation of potential sources of information. This factor overcomes one of the major imitations of the general case study approach, the challenge of obtaining complete information. As with all 
investigation of past events, the forensic case study, like the general case study, can only obtain information which was created in the performance of the activity under investigation, and that information is only what was required by the processes in place while the events were unfolding.

Forensic investigation for the purpose of identifying responsibility for a state of affairs will lead to a clear account of what happened in the case. That knowledge is not, itself, guidance for what should to be done in future cases. In contrast, a coronial inquest or a commission of inquiry, are type of investigation intended to find out what happened and to interpret the information in a way that leads to recommendations for approaches that will reduce the risk of similar bad events in the future. The case study performed to generate recommendations for future action demands investigation of the emergent effects of the factors present in the case and also, investigation of the emergent effects that it would be reasonable to expect in the event of implementation of any recommendation.

\section{Post Hoc Empirical Studies}

Post hoc empirical studies involve observation of one or more cases with the purpose of drawing conclusions about the situation of which the cases are believed to be representative. However, the investigator is limited to observing the situation through data that has been collected and divulged to the investigator about cases over which the investigator has had no control.

In this kind of study the investigator identifies a population of existing cases which fit criteria of interest to the investigator and then seeks to analyse data arising from those cases with the purpose of making a broader conclusion. This approach to empirical research is often taken if there are practical, ethical or safety difficulties with performing an experiment in which the investigator applies certain treatments or non-treatments to sub-groups of the population under study with a view to testing whether those treatments have distinguishable correlation with an outcome manifestation.

There are multiple challenges with the post hoc empirical study. First, the cases from which the study sample is obtained pre-exist the study. The effect is that separation of the cases of interest is from other factors is precluded, making all observations in situ, not allowing a separation of the effect of the matter of interest from other known or unknown effects.

A second effect of studying pre-existing cases is that there can be no randomisation of the cases receiving each of the treatments, but rather the cases exist and were given the particular treatment they received because someone managing the case believed, a priori, the particular treatment applied to be the way to improve the probability of achieving their desired outcome. Thus, all combinations of cases and treatments are biased by the belief that the combination was a good thing, precluding conclusions about what may happen in circumstances where the actor has no a priori basis for believing a certain treatment to be appropriate.

A third effect of the pre-existing nature of the cases investigated is that the data collected to describe the case and the action performed upon it organises the representation of the situation according to categories chosen, or accepted by those responsible for the case. The data could be further biased by the quality of the measurement or observation processes used as means of providing accurate data of the situation.

A fourth problematic factor in the post hoc study is the choice of cases that the investigator makes although a careful and honest investigator would attempt to choose cases that are representative of the population of interest. However, it is possible, for a variety of reasons, that the sample may reflect a bias towards one sub-group in the population.

In summary, the post hoc study is likely to struggle to provide insight into the relationship in the study space that lead to the generation of the emergent effects which may have motivated the study. The challenge may arise at several levels: the difficulty in obtaining sufficient data to make a judgment of correlation of observable manifestations, and the absence of control for other factors makes the deduction or refutation of causality invalid.

\section{Experimental Empirical Studies}

Experimental studies differ from post hoc studies in the fundamental matter that the investigator is able to set up the conditions to which the cases are exposed and select which cases are assigned to which condition in order to enable a comparison of case, input conditions applied and observed outcome. There are well established protocols for randomly assigning cases to treatments and, to the greatest extent possible, blinding the investigator and any other participants in the experiment to the correlation of cases and treatment in order to reduce the risk of observation bias in relation to description, where heuristic representation of effects is used, or measurement bias, in either case weighting the record of observation to favour the observer's preferred outcome.

The challenge, in relation to emergence, of experiments is that by nature, an experiment is performed to observe a statistically significant number of cases in order to present results that enable a conclusion that the effect is, or is not, statistically significant. As such, substantial numbers of cases must be investigated in order to provide statistical assurance of difference in outcome as a function of the treatment applied. Therefore, it is common to conduct experiments to investigate the relationship of an independent and a dependant variable in a particular type of entity in association with a set of environmental conditions, each at a set value. The effect of this is that it is a very large task to set up a set of conditions that would explore emergent effects of a large number of factors, each of which could have an influence on the observed behaviour of the entity under test.

A consequence of this is that a relatively small number of experimental tests are conducted on whole systems. Examples include, in the motor vehicle sector, crash tests, field consumption and emissions tests. In each of these cases the tests are conducted against a standard that describes the method of the test and exact parameters of a range of operational and environmental factors, and also, if relevant, the observed outcomes for pass or fail criteria. Because these experiments, which do test the emergent characteristics of the whole system, 
are expensive to perform it is normal for the standard to define a small number of tests of distinctly different scenarios which are posited as representative of situations that may be encountered.

There is no empirical test of response of the system to similar but different conditions, that is, any attempt at sensitivity analysis will only be performed in the simulation space, not the hard empirical space. The effect is that it is possible to design for the exact tests to which the system is required to be subjected with a lack of attention to sensitivity analysis to the variety of cases of which the test standard is presumed to be representative.

Experimental studies are designed for, and well suited to, the investigation of relationships of phenomena but are poorly suited for both resource and combinatorial complexity reasons to the investigation of system behaviour across the whole of the operation envelope.

\section{E. Research that Enables Engineering}

The approaches to research described above are useful for discovery of facts and interpretations about either particular cases, in the case study and forensic case study scenarios, or learning about phenomena in either of the empirical scenarios. This presents a challenge for engineers and engineering. Engineering is concerned with the application of all the available knowledge to enable taking action that provides desired effects, and simultaneously avoids undesirable effects.

The engineering need is for knowledge of a different kind than is typically developed in the methods described above. The previous methods are either historical, investigating existing cases, or oriented to science, in which the discovery of the knowledge is valued for the presumed value of the knowledge, which does inform engineering work, but through a somewhat indirect path, which is, because of the effect of emergence, fraught with risk that the designs recommended may manifest unexpected emergent effects.

To generate knowledge that is useful in engineering a wide range of methods may be used. The methods described in the other subsections of this section of the paper are all focused on development of knowledge about things, phenomena or relationships which may be useful in the development of engineered technologies. The conduct of engineering activity uses other knowledge about methods and process, both related to the tangible products of the engineering and also the ways and means of performing the engineering activities. Discovery of these things may require the use of further research methods and approaches not discussed in this paper. The approaches to research discussed in this paper were selected as the approaches which are most likely to inform about emergence in some way and are commonly used in engineering research.

\section{F. Research into Fundamental Theory}

This research is performed in the space of abstractions in order to determine the properties of the models used to describe situations of interest in the theory of the field. This research is usually performed in the mathematical representation space, relying on the methods of mathematics and the observed homomorphism of mathematics and the physical phenomena represented.

The mathematical methods, taken alone, follow methods such as the proof of theorems, or the implementation of numerical modelling within the mathematical space. The outcome of such studies, in a strict sense, informs about the properties of the models constructed of the systems, rather than of the systems themselves. This distinction is important because it is the source of possible difference between results predicted through use of the models and results observed when the systems themselves are implemented and operated.

Research using the fundamental theory methods might find some emergent effects. The class of emergent effect which mathematics can discover is the consequences of things which are already known but where the consequences are not yet known because they arise through the complexity of interaction of the elements which are known. This class of discoverable emergence fit the philosophical question discussed in section II. This type of emergence is used powerfully in engineering design because the computational power now available, combined with the scientific knowledge of phenomena of interest for application in engineering because, through the modelling of multiple design alternatives it is possible to identify particular choices that generate useful results that are unlikely to be found through less computationally intensive methods.

However, the use of mathematical modelling methods, based on fundamental theory, is unable to discover emergent effects arising from either the combination of physical phenomena for which there is not yet a theory, or even empirical results, which could be incorporated into a model. In addition, mathematical modelling methods are inherently limited to exploration of the effects which have been incorporated into the model. Therefore, if there is a relevant effect which could generate emergent effects, but it is not incorporated into the model then fundamental theory based methods of investigation cannot find the emergent effect resulting.

\section{DISCUSSION OF THE RESEACH GAP: ENGINEERING}

In section III we outlined six methods of investigation commonly used in association with engineering with particular interest in the relationship of those methods and engineering. The methods are used in other fields and any statements of limitations of the methods above is to be understood as relating to the approach to research as a tool for assisting engineering work.

Engineering is fundamentally different than science because engineering is concerned with performing appropriate action or delivering appropriate solutions to practical needs, in contrast to finding out about things. As such, the matter of emergence is the core space of engineering, which is interested in assembling things so that desirable effects different than what is observed in the parts is what is desired. Therefore, engineers need means of addressing the question of emergence systemically to enable the discovery of emergent effects beyond those that are discoverable through the design modelling work normally performed in design, which is limited 
to the effects that the engineer thinks of including in an analysis.

The gap for which a solution is needed is how to predict emergent effects which will become evident upon construction of the system as designed. This has two parts:

1. Prediction of emergent effects arising from known relationships of phenomena. The system design involves things and relies on phenomena that are understood. The knowledge of these things is what prompts the designer to propose the system design and in many cases awareness of particular pathways of emergent effects may inspire a design strategy. These effects can be modelled because the knowledge of the relationships embedded in them is explicit.

2. Prediction of emergent effects of kinds not previously known. This class of effect is more difficult. The challenge is that the specific interactions are not known and therefore there is no previous work available that has found the kind and magnitude of the effects. Therefore, it is necessary to posit possible linkages that could cause emergent effects. This could arise through any possible emanation from each item in the system, which are the causative means, and any possible input. The means of interaction between the elements of a system could be of any of the forms: energy, matter, material wealth and information. These things are the means by which the communication between system elements that could instantiate emergence. If a possible interaction is identified this leads to a need to review prior research to determine if there is existing knowledge of the relationship. In the absence of prior knowledge of the relationship it is essential to perform new research to investigate the possible relationship. Results of such research, newly performed or found, can then be incorporated into models of the proposed system, as in 1. above. It is to be noted that each of the possible forms of interaction could exist in a variety of actual forms.

\section{CONCLUSIONS}

Emergence is a fundamental part of the engineering enterprise. Existing methods of research provide means of discovering either the properties and relationships of things and the history of emergent interactive effects. This knowledge is helpful in enabling prediction and analysis of possible interactions which could lead to emergent effects in systems. The challenge is that many interactive effects may not be known as existing, or described in a manner that enables prediction of effects. This paper describes the problem and identifies an approach which can lead to the prediction of emergent effects, thus reducing the risk associated with unexpected emergence.

\section{REFERENCES}

[1] E. L. Gettier, "Is justified true belief knowledge?," in Knowledge and belief, A. P. Griffiths, Ed. London: Oxford University Press, 1967, pp. 144-146.

[2] J. Hintikka, "Knowledge, belief, and logical consequence," Ajatus, vol. 32, pp. 32-47, 1970.

[3] N. Goodman, Fact, fiction, and forecast. Indianapolis, Indiana: The Bobbs-Merrill Company, Inc., 1973.

[4] P. Checkland and S. Holwell, Information, systems and information systems. Chichester, England: Wiley, 1998.

[5] S. C. Cook and T. L. J. Ferris, "Re-evaluating systems engineering as a framework for tackling systems issues," Syst. Res. Behav. Sci., vol. 24, pp. 169-181, 2007.

[6] D. Rousseau, J. Billingham, J. Wilby, and S. Blachfellner, "In search of General Systems Theory," Systema, vol. 4, no. 1, pp. 7699, 2016. 